\title{
UWB Impulse Radio with Triple-Polarization SIMO
}

\author{
Wasim Q. Malik and David J. Edwards \\ Department of Engineering Science, University of Oxford \\ Parks Road, Oxford OX1 3PJ, United Kingdom \\ Email:wasim.malik@eng.ox.ac.uk, david.edwards@eng.ox.ac.uk
}

\begin{abstract}
Polarized single-input multiple-output (SIMO) arrays can improve system robustness and rate while offering a compact design. We investigate the error performance of Impulse Radio ultrawideband (UWB) systems with multipleantenna arrays employing polarization orthogonality. Based on indoor channel measurements, we evaluate the improvement in the link reliability due to dual- or triple-polar antenna arrays. Using a two-dimensional combining receiver, it is demonstrated that polarization diversity can be very effective in reducing the overall system complexity, making Impulse Radio implementation practically feasible.
\end{abstract}

\section{INTRODUCTION}

Ultrawideband (UWB) technology is a leading contender for future wireless applications such as multimedia communications, sensor networks and imaging systems [1]. A major limitation of UWB systems is the limited range, a result of the regulatory constraints on the transmit power levels. Another consequence of the power limits is the performance degradation in highly cluttered environments, such as those represented by the IEEE 802.15.3a CM4 channel model [1].

Diversity reception is a well established technique for improving the system performance and mitigating the fading under harsh propagation conditions. Wideband, frequencyselective channels can make use of rake reception as a multipath diversity mechanism [2]. Some UWB implementations, such as those based on direct-sequence spread-spectrum or time-hopping, exemplify this regime. A particulary important and popular implementation is the Time-Hopping Impulse Radio scheme, first proposed by Win and Scholtz [3], that has recently been adopted by IEEE $802.15 .4 \mathrm{a}$ for its wireless sensor networks standards [1]. Given the fine time resolution and dense multipath propagation in the typical UWB channel [4], the Impulse Radio scheme involves a considerably complex rake receiver structure for appreciable energy capture.

The use of multiple antennas provides an alternative form of diversity. Multiple-antenna systems can use the independence of signal fading in the spatial, angular, or polarization domain to improve the performance. While spatial diversity is most common and well understood, spatial arrays require a certain minimum inter-element spacing for the diversity gain to be realized, which is on the order of half-wavelength for uniform linear arrays (ULAs). The analysis in [5] shows that an interelement spacing of $6 \mathrm{~cm}$ is sufficient to achieve uncorrelated fading on the diversity branches of a multiple-antenna UWB system. This spacing, is however, considerably large when some typical UWB applications, such as USB interfaces,
PCMCIA cards, handheld devices and sensor motes, are considered, as the surface area on these devices generally cannot accommodate a linear array of several such elements.

Polarization arrays can offer a solution to this problem, as three orthogonally polarized elements can be collocated and yet be used for signal extraction with uncorrelated fading, provided dense multipath conditions. For this reason, polarization arrays have attracted great interest in recent years for diversity and multiplexing applications [6]-[9]. In our earlier work, we have analyzed the capacity and SNR improvement provided by polarized MIMO antennas in indoor UWB channels, and found it to approach that of spatial MIMO especially in non-lineof-sight (NLOS) environments, establishing the value of the technique [5]. We have also designed a collocated tri-polarized array with planar construction, which makes it possible to fabricate and implement a triple-polarized antenna structure even within a compact system such as handheld devices and wireless network cards for computer communications [10].

In their recent work, Keignart et al. pointed out the possibility of using UWB antenna diversity to offset the rake complexity [11]. Bit-error rate (BER) simulations of the measured SIMO channel showed that an $N$-element ULA with a singlefinger rake is more effective than a single-antenna system with an $N$-finger rake in achieving higher system robustness. Using a simulated indoor channel, Argenti et al. found dualpolar diversity helpful in maintaining constant quality of service irrespective of the instantaneous antenna orientation in multiband UWB systems [12]. Recognizing the importance of polar arrays, we extend the analysis of polarization diversity to Impulse Radio with dual- and tri-element SIMO arrays in this paper. We consider the BER performance of the system, obtained from UWB SIMO channel measurements conducted in indoor office environments over the FCC-allocated UWB band (3.1-10.6 GHz). The proposed UWB vector rake receiver exploits signal diversity simultaneously in the multipath and polarization dimensions to enhance the system robustness.

\section{SYSTEM MODEL}

We consider an Impulse Radio UWB system with $1 \times N$ SIMO antenna configuration, operating in an indoor multipath channel, as shown in Fig. 1. The multipath-dispersed and polarization-rotated signal incident at the $n^{\text {th }}$ antenna is

$$
y_{n}(t)=\sqrt{E_{s}} h_{n}(\tau) * s(t)+z_{n}(t)
$$

where $E_{s}$ is the transmit symbol energy, $s(t)$ is the transmit signal such that $|s(t)|^{2}=1$, and $z_{n}(t)$ is the additive white 

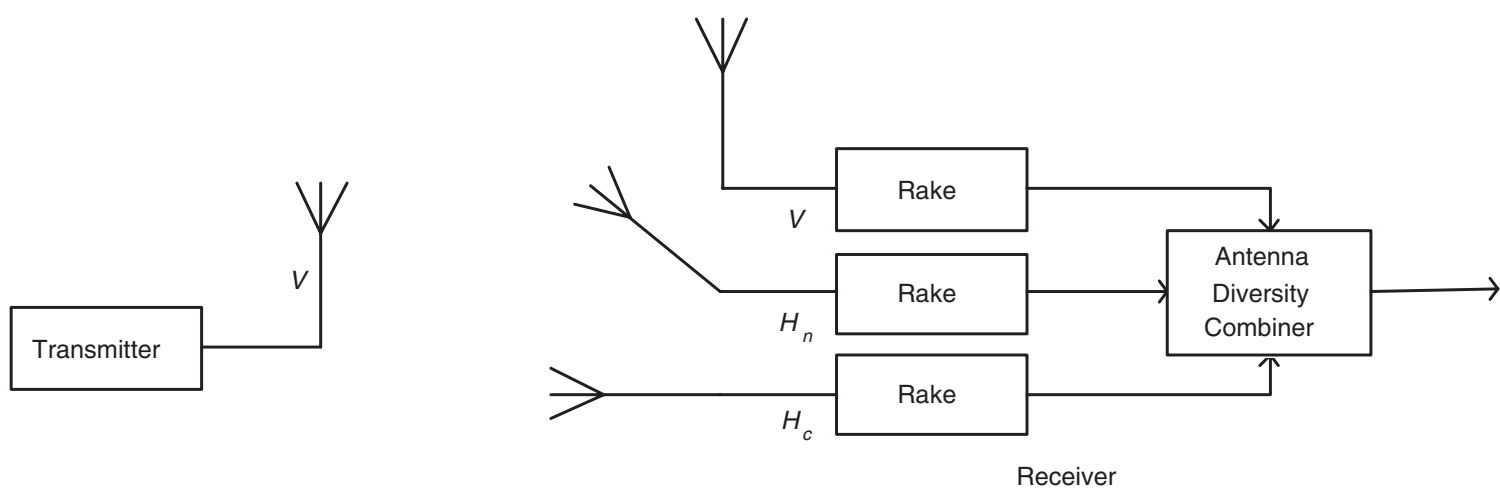

Fig. 1. The system architecture for the Impulse Radio single-input multiple output (SIMO) system using a linear triple-polarization antenna array. The mutually perpendicular antenna elements $V$ and $H_{n}$ are in the plane perpendicular to the Poyting vector of the direct path, while $H_{c}$ is collinear with it.

Gaussian noise with variance $N_{0}$. The above expression is the system model for the $n^{\text {th }}$ constituent single-input single-output (SISO) link, where $n \in\{1, \ldots, N\}$. In $(1), h_{n}(\tau)$ is a given realization of the UWB channel impulse response (CIR) at the $n^{\text {th }}$ diversity branch, given by the tapped delay line model as

$$
h_{n}(\tau)=\sum_{l=1}^{L_{n}} \alpha_{l, n} \delta\left(\tau-\tau_{l, n}\right),
$$

where $L_{n}$ is the number of resolved multipath components (MPCs), $\tau$ is the time delay with respect to the first multipath arrival, and $\alpha_{l, n}$ and $\tau_{l, n}$ represent the amplitude and time delay of the $l^{\text {th }}$ MPC. The SIMO channel can be represented by $\mathbf{h}(\tau)=\left[h_{1}(\tau), \ldots, h_{N}(\tau)\right]^{\mathrm{T}}$, where $(.)^{\mathrm{T}}$ represents the transposition operation. The $V-V$ channel, $h_{1}$, is designated as the reference diversity branch and the SIMO channel, $\mathbf{h}(\tau)$, is normalized such that $\int_{\tau=0}^{\infty}\left|h_{1}(\tau)\right|^{2} \mathrm{~d} t=\sum_{l=1}^{L_{1}}\left|\alpha_{l, 1}\right|^{2}=1$.

The received signal, $y_{n}(t)$, at the $n^{\text {th }}$ receive branch is fed to a rake receiver for multipath combining. We consider hybridselection/maximal-ratio combining (H-S/MRC) rake reception, also known as the selective rake scheme [2]. The rake receiver consists of $M$ fingers, so that it captures $M$ of the $L_{n}$ strongest MPCs, where $M \leq L_{n}$. MRC is the optimal combining scheme for interfere-free multipath channels and therefore provides a realistic upper bound on the rake performance, whilst the subset selection reduces the complexity to the desired level. Thus, the resolved MPCs are ordered with respect to their SNRs prior to combining, with their amplitudes given by $\alpha_{m, n}$, where $m \in\{1, \ldots, M\}$ is the index to the sequence of selected MPCs in descending order of SNR. Correspondingly, the path SNR is given by $\gamma_{m, n}=\left|\alpha_{m, n}\right|^{2} / N_{0}$, such that $\gamma_{1, n} \geq \ldots \geq \gamma_{M, n}$. As the mean SNRs of the MPCs are, in general, unequal, the $M$ MPCs are differentially emphasized with weights $w_{m, n}$ such that $w_{m, n} \propto\left|\alpha_{m, n}\right|^{2}$ and $\sum_{m=1}^{M} w_{m, n}=1$. After multipath co-phasing and addition at the rake in the receive chain corresponding to the $n^{\text {th }}$ antenna, the SNR of its output signal, $r_{n}$, can be written as

$$
\gamma_{M, n}^{\prime}=\sum_{m=1}^{M} \gamma_{m, n} .
$$

The rake outputs are then fed to the antenna diversity combiner, which uses the MRC scheme to combine the signals received at the $N$ orthogonally polarized antenna elements, where $N \in \mathbb{Z}^{+}, N \leq 3$. We consider the general case that the three polarization branches have unequal branch power ratios, as was indeed established in [5]. Taking into account the unequal branch SNRs, the weight $v_{n}$ is applied to the $n^{\text {th }}$ branch of the antenna combiner such that $v_{n} \propto \gamma_{M, n}^{\prime}$ and $\sum_{n=1}^{N} v_{n}=1$. The SNR of the signal, $r$, obtained at the output of the antenna combiner can then be expressed as

$$
\Gamma_{M, N}=\sum_{n=1}^{N} \gamma_{M, n}^{\prime}=\sum_{n=1}^{N} \sum_{m=1}^{M} \gamma_{m, n} .
$$

The signal $r$ is used for symbol demodulation and detection in the following stages of the receiver. It is obvious from the right-hand side of (4) that the order of combining in Fig. 1 can be switched, i.e., antenna combining can be performed before rake combining, without affecting the analytical formulation.

\section{Performance Evaluation}

In this section, we evaluate the performance of the proposed Impulse Radio system with polarization diversity. We first describe the channel measurement procedure to obtain the UWB SIMO channel realizations used for the bit-error rate (BER) analysis, followed by some of the key results.

\section{A. Channel Measurements}

SIMO channel measurements are conducted over the UWB spectral range, 3.1-10.6 GHz, as allocated by the FCC for indoor communications. A vector network analyzer is used to sample the complex $S_{21}(f)$ parameter at $F=1601$ discrete, uniformly spaced frequency points within the spectral range of interest with bandwidth $W=7.5 \mathrm{GHz}$. The measurement system is calibrated to remove the frequency-selective distortion due to the cables, connectors and amplifiers. Discone antennas, with omni-directional radiation patterns, are used to perform the measurements. The antennas are rotated to obtain the three orthogonal linear polarization configurations while keeping the physical location constant, synthesizing a collocated array of polarized antennas. This configuration is referred to as a 

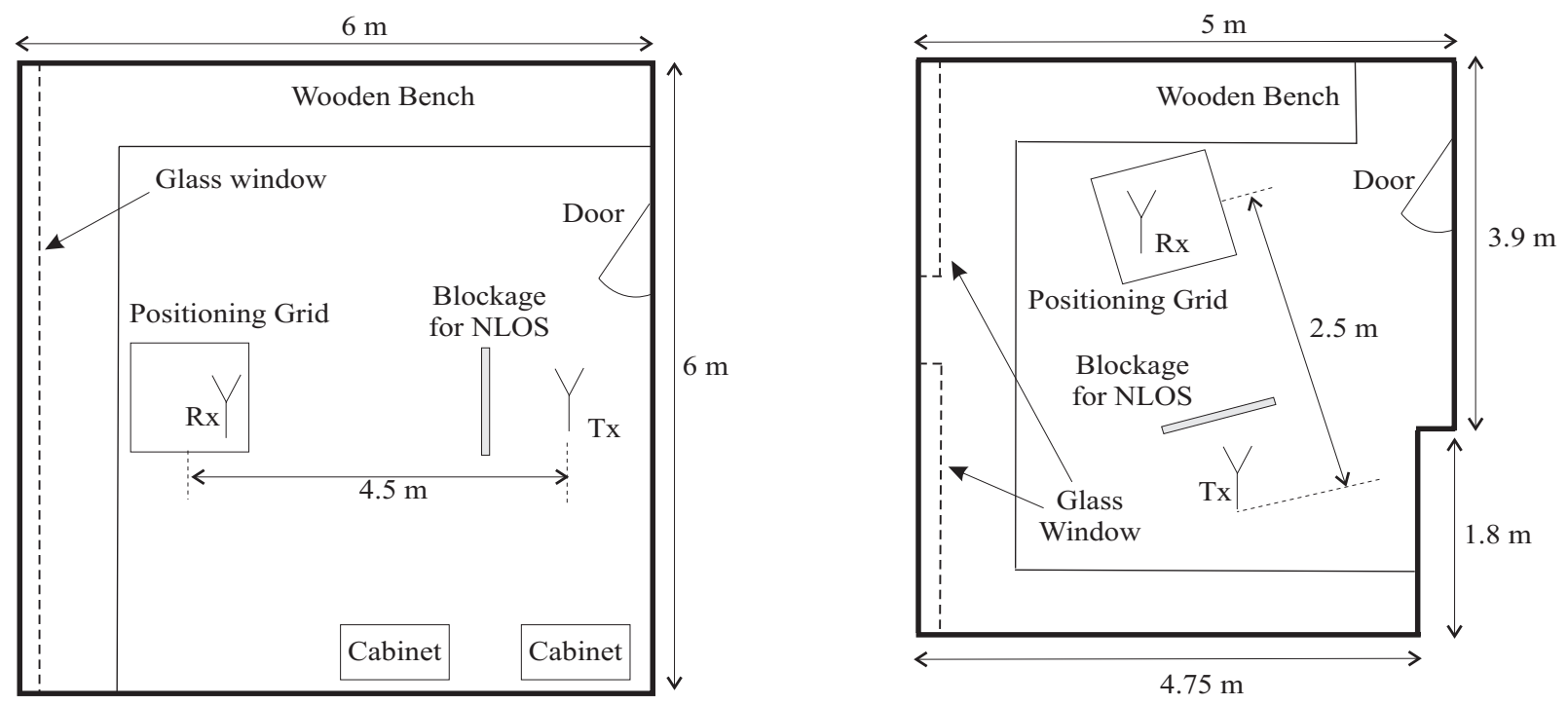

Fig. 2. The measurement environment for UWB channel measurements with triple-polarized SIMO diversity arrays.

virtual array, making use of sequential measurements under the condition of channel stationarity. The polarization diversity obtained in this manner also contains the effect of pattern diversity as an inevitable by-product of this approach [5].

The environments consists of two separate medium-sized office rooms with a typical amount of multipath clutter present, as shown in Fig. 2. Line-of-sight (LOS) and non-LOS (NLOS) measurements are conducted in each case. The environment is kept static throughout the duration of the measurement by ensuring that there is no mobility in the surroundings. At each site, an automated receiver positioning system is used to obtain the channel measurements over a $1 \mathrm{~m}^{2}$ region with a spatial resolution of $0.03 \mathrm{~m}$. A set of measured UWB SIMO channel realizations with ensemble size $R=1800$ is thus obtained. For further details of the measurement procedure, see [5].

The measured channel responses are converted to the time domain using the discrete inverse Fourier transform. A $20 \mathrm{~dB}$ power threshold is then applied to remove the noise and residual multipath, followed by MPC detection based on local maxima search across the discrete time-bins. The propagation delay of the first MPC is removed to obtain the CIRs, $\mathbf{h}_{r}(\tau)$.

\section{B. Error-Rate Analysis}

We now use the system BER as a means to compare the performance obtained with various rake and antenna diversity configurations. For the error-rate comparisons in this paper, we consider binary antipodal modulation using the binary phase shift keying (BPSK) scheme. The probability of bit error for this modulation scheme, conditioned on the $r^{\text {th }}$ SIMO channel realization, $\mathbf{h}_{r}$, is given by [11], [12]

$$
P_{e}\left(\mathbf{h}_{r}\right)=Q\left(\sqrt{2 \rho \Gamma_{M, N}}\right),
$$

where $\rho=E_{s} / N_{0}$ is the transmit SNR, $\Gamma_{M, N}$ is the output SNR of the diversity system corresponding to the channel $\mathbf{h}_{r}$, and $Q($.$) is the Marcum Q-function. For a given \rho$, we evaluate $P_{e}$ for each channel realization. The average BER is obtained as the expectation over the ensemble with $R$ realizations, i.e.,

$$
\bar{P}_{e}=\frac{1}{R} \sum_{r=1}^{R} P_{e}\left(\mathbf{h}_{r}\right) .
$$

In the subsequent discussion, we consider the following polarization diversity array configurations:

(1) $N=1$ refers to the $V-V$ SISO system;

(2) $N=2$ refers to the $V-V H_{n}$ SIMO system; and

(3) $N=3$ refers to the $V-V H_{n} H_{c}$ SIMO system.

Fig. 3 shows the variation of $\bar{P}_{e}$ with the transmit SNR, $\rho$, for the two extreme values of $M$, i.e. $M=1$ (selectioncombining rake with one finger) and $M=L$ (ideal maximalratio combining or all-rake with sufficient fingers to capture all paths). For given $M$, we can observe the negligibly small improvement in the diversity order, $d=-\lim _{\rho \rightarrow \infty} \bar{P}_{e} / \rho$, with increase in $N$. This is especially true in the LOS channel and for large $M$, where SIMO cannot increase $d$ since most of the available diversity in the channel has already been extracted. The difference in the LOS and NLOS $d$ behavior is due to the lower cross-polar discrimination (XPD) and branch power ratio of the $V V$ link in NLOS, caused by the direct path blockage and multiple scattering, so that polarization diversity captures a greater portion of the incident signal power.

Compared to the diversity order improvement, however, a more noticeable result of polarization diversity is the overall SNR gain at the receiver, quantified in terms of the reduced transmit SNR, $\rho$, for a given $M$ required to achieve a certain $\bar{P}_{e}$ threshold, as observed in Fig. 3. Tri-polar diversity thus improves the performance of a single-finger rake system by $2 \mathrm{~dB}$ and $4.5 \mathrm{~dB}$ in LOS and NLOS, respectively. The higher gain in the NLOS channel is due to reduced power imbalance across the branches because of lower XPD. In both LOS and 


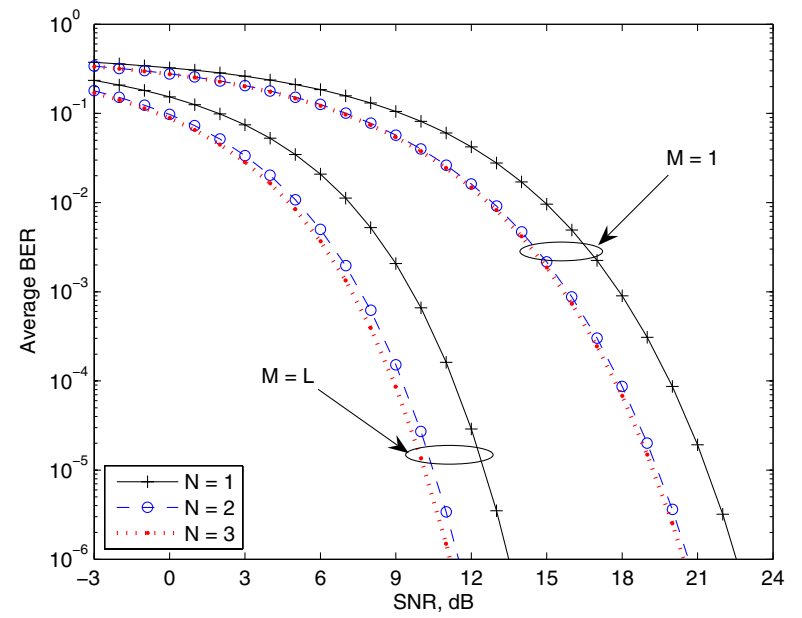

(a) Line-of-sight channel

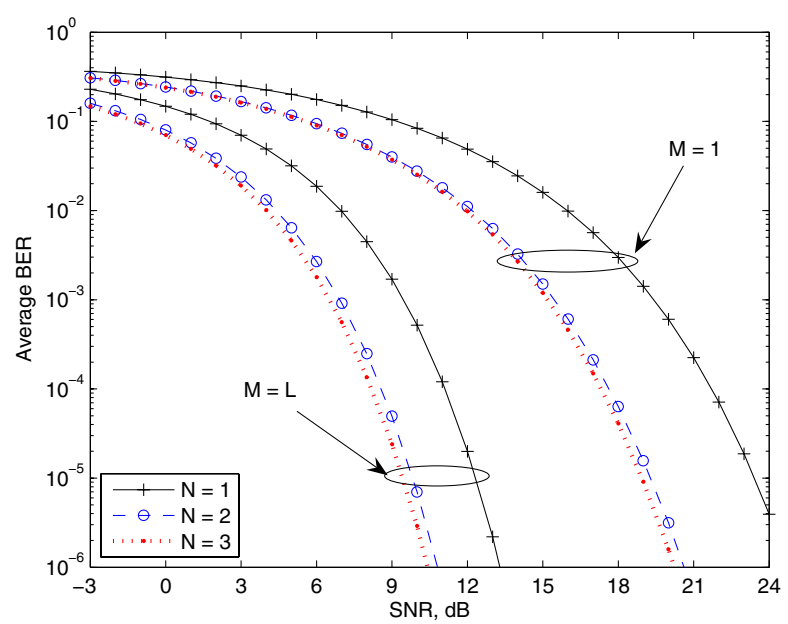

(b) Non-line-of-sight channel

Fig. 3. The average bit-error rate (BER) of the $1 \times N$ polarization diversity Impulse Radio system with $M$ rake fingers and $L$ multipath components.

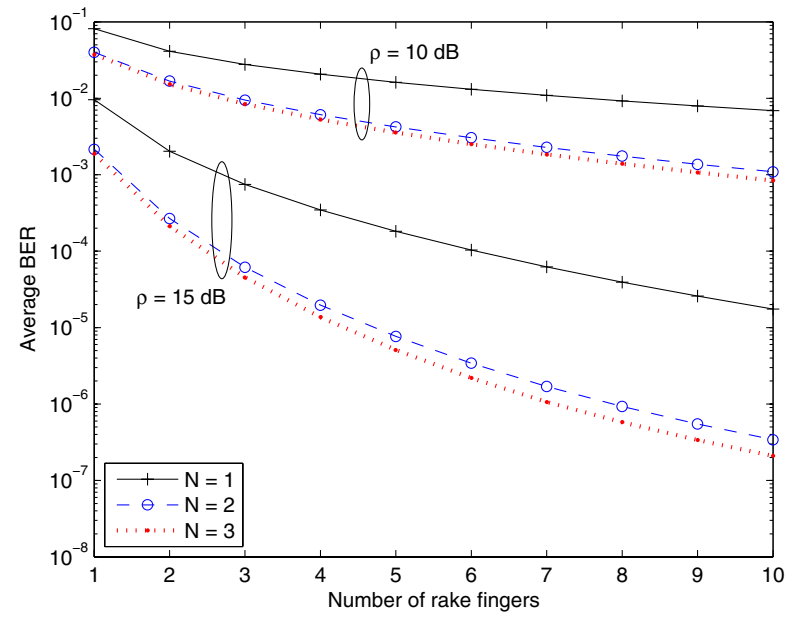

(a) Line-of-sight channel

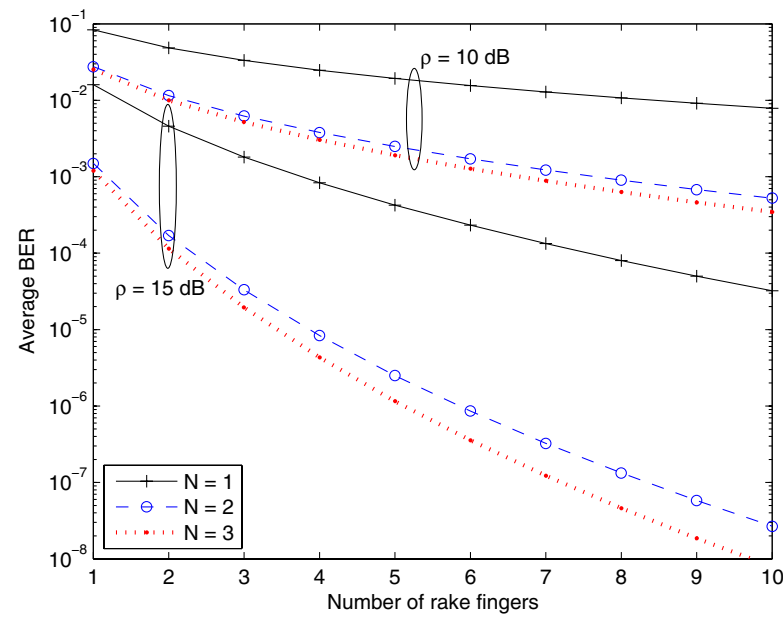

(b) Non-line-of-sight channel

Fig. 4. The average bit-error rate (BER) of the $1 \times N$ polarization diversity Impulse Radio system at SNR $\rho$.

NLOS, the contribution of the $H_{c}$ branch to the diversity system is quite low, which is due to the low branch power ratio of the $V-H_{c}$ link, a consequence of the fact that $V-H_{c}$ propagation relies exclusively on substantial signal depolarization from multiple scattering events [5], [7].

The detailed analysis in Table I reveals that polarization diversity is sometimes more effective than multipath (rake) diversity in overcoming fading and reducing the required transmit energy. For example, we find that in NLOS, the performance of a single-finger dual-polar system is similar to that of a tri-finger single-polar system. These observations are consistent with those reported for an Impulse Radio system using spatial antenna diversity in [11]. Thus it is noticed that polarized antenna elements can be used as a replacement for rake fingers, offering a complexity tradeoff for diversity between the multipath and polarization domains.

Fig. 4 shows the behavior of $\bar{P}_{e}$ as a function of $M$ for some selected values of $\rho$. At $\rho=15 \mathrm{~dB}, \bar{P}_{e}=10^{-4}$ can be achieved by a $1 \times 2$ SIMO system with $M=3$, while a $1 \times 1$ SISO system requires $M=6$ and 8 in LOS and NLOS, respectively. The reduction in the required rake receiver complexity due to polarization diversity is evident.

We can represent the overall system complexity of the twodimensional diversity system by $T=N+M$. The results of our analysis show that in many cases, for a given $T$, the $\bar{P}_{e}$ is lower for constant $\rho$, or alternatively $\rho$ is lower for constant $\bar{P}_{e}$, when $N>M$ and $T$ has a realistic value. This is especially true for propagation environments dense scattering such as the non-line-of-sight indoor channel. In other words, compared to a conventional Impulse Radio system, the proposed vector rake 
TABLE I

SNR (IN DB) REQUIRED TO ACHIEVE $\bar{P}_{e}=10^{-4}$ WITH $1 \times N$ SIMO

\begin{tabular}{ccccccc}
\hline \hline \multirow{2}{*}{$\begin{array}{c}\text { Rake } \\
\text { fingers }\end{array}$} & \multicolumn{3}{c}{ Line-of-sight channel } & \multicolumn{3}{c}{ Non-line-of-sight channel } \\
\cline { 2 - 6 } & $N=1$ & $N=2$ & $N=3$ & $N=1$ & $N=2$ & $N=3$ \\
\hline 1 & 19.8 & 17.9 & 17.7 & 21.7 & 17.6 & 17.3 \\
2 & 17.7 & 15.8 & 15.6 & 19.1 & 15.4 & 15.1 \\
3 & 16.6 & 14.7 & 14.4 & 17.7 & 14.2 & 13.9 \\
4 & 15.9 & 14.0 & 13.7 & 16.8 & 13.4 & 13.1 \\
5 & 15.4 & 13.4 & 13.2 & 16.0 & 12.9 & 12.5 \\
10 & 13.9 & 11.9 & 11.6 & 14.3 & 11.2 & 10.9 \\
$L$ & 11.3 & 9.2 & 8.9 & 11.1 & 8.6 & 8.2 \\
\hline \hline
\end{tabular}

scheme with polarized antenna diversity can reduce the overall system complexity or enhance the performance significantly.

\section{CONCLUSION}

In this paper, we have studied the error performance of the Impulse Radio UWB system with polarized SIMO arrays operating in measured indoor channels. We have shown that collocated polarized arrays can provide considerable antenna diversity and improve the link performance both in LOS and NLOS environments. The diversity system can be used to enhance the coverage range and data-rates, or to reduce the transmit power and therefore the potential interference to other systems. The gain of a tri-polar system over a dual-polar system is only up to $0.5 \mathrm{~dB}$, due to the very low branch power ratio associated with the $V-H_{c}$ cross-polarized channel. It has been demonstrated that a polarization array at the receiver can significantly reduce the number of required rake fingers. The overall complexity of the two-dimensional diversity system has been shown to be lower than that of the conventional rake scheme, and the proposed technique can therefore be immensely useful in practical UWB system design.

\section{ACKNOWLEDGMENT}

The authors are grateful to Athanasios Kavatjikidis and Matthews C. Mtumbuka for assisting with the channel measurements. The financial support from the UK Engineering and Physical Sciences Research Council via Grant GR/T21769/01 is also gratefully acknowledged.

\section{REFERENCES}

[1] B. Allen, M. Dohler, E. E. Okon, W. Q. Malik, A. K. Brown, and D. J. Edwards, Eds., Ultra-Wideband Antennas and Propagation for Communications, Radar and Imaging. London, UK: Wiley, 2006.

[2] M. Z. Win, G. Chrisikos, and N. R. Sollenberger, "Performance of rake reception in dense multipath channels: implications of spreading bandwidth and selection diversity order," IEEE J. Select. Areas Commun., vol. 18, no. 8, Aug. 2000.

[3] M. Z. Win and R. A. Scholtz, "Impulse radio: how it works," IEEE Commun. Lett., vol. 2, no. 2, Feb. 1998.

[4] A. F. Molisch, "Ultrawideband propagation channels - theory, measurement, and modeling," IEEE Trans. Veh. Technol., vol. 54, no. 5, Sept. 2005.

[5] W. Q. Malik and D. J. Edwards, "Measured MIMO capacity and diversity gain with spatial and polar arrays in ultrawideband channels," IEEE Trans. Commun., in press.

[6] R. G. Vaughan, "Polarization diversity in mobile communications," IEEE Trans. Veh. Technol., vol. 39, no. 3, Aug. 1990.

[7] M. R. Andrews, P. P. Mitra, and R. deCarvalho, "Tripling the capacity of wireless communications using electromagnetic polarization," Nature, vol. 409, Jan 2001

[8] S. Kozono, T. Tsuruhara, and M. Sakamoto, "Base station polarization diversity reception for mobile radio," IEEE Trans. Veh. Technol., vol. VT-33, no. 4, Nov 1984.

[9] M. Shafi, et al., "Polarized MIMO channels in 3D: models, measurements and mutual information," IEEE J. Select. Areas Commun., vol. 24, no. 3, Mar. 2006.

[10] D. J. Edwards, T. Hao, W. Q. Malik, and C. J. Stevens, "A planar tripolar antenna," in British Patent (pending). University of Oxford, 2007.

[11] J. Keignart, C. Abou-Rjeily, C. Delaveaud, and N. Daniele, "UWB SIMO channel measurements and simulations," IEEE Trans. Microwave Theory Tech., vol. 54, no. 4, Apr. 2006.

[12] F. Argenti, T. Bianchi, L. Mucchi, and L. S. Ronga, "Polarization diversity for multiband UWB systems," Sig. Proc., vol. 86, no. 9, Sept. 2006. 